02,03

\title{
Сверхпроводимость в тонких пленках дираковского полуметалла $\mathrm{Cd}_{3} \mathrm{As}_{2}$
}

\author{
() А.Б. Давыдов ${ }^{1}$, Л.Н. Овешников ${ }^{1,2}$, А.В. Суслов ${ }^{3}$, А.И. Риль ${ }^{4}$, С.Ф. Маренкин ${ }^{4,5}$, Б.А. Аронзон ${ }^{1}$ \\ ${ }^{1}$ Физический институт им. П.Н. Лебедева РАН, \\ Москва, Россия \\ ${ }^{2}$ НИЦ „Курчатовский институт“, \\ Москва, Россия \\ ${ }^{3}$ National High Magnetic Field Laboratory, Tallahassee, \\ Florida, USA \\ ${ }^{4}$ Институт общей и неорганической химии им. Н.С. Курнакова РАН, \\ Москва, Россия \\ ${ }^{5}$ Национальный исследовательский технологический университет „МИСиС“ \\ Москва, Россия \\ E-mail: sanyadav@yandex.ru
}

Поступила в Редакцию 28 октября 2019 г.

В окончательной редакции 28 октября 2019 г.

Принята к публикации 31 ноября 2019 г.

Исследовались тонкие пленки дираковского полуметалла $\mathrm{Cd}_{3} \mathrm{As}_{2}$, толщиной около $100 \mathrm{~nm}$, изготовленные с помощью вакуумно-термического напыления из монокристаллов арсенида кадмия, приготовленных непосредственным сплавлением элементов вакуумно-ампульным методом. Были проведены измерения температурных и магнетополевых зависимостей электросопротивления, которые свидетельствуют о наличии сверхпроводящего перехода при температуре от 0.2 до $0.6 \mathrm{~K}$.

Ключевые слова: Дираковский полуметалл, сверхпроводимость, тонкие пленки.

DOI: 10.21883/FTT.2020.03.48998.617

Настоящая работа посвящена экспериментальному наблюдению сверхпроводимости в дираковском полуметалле $\mathrm{Cd}_{3} \mathrm{As}_{2}$ в тонких пленках с толщиной $\sim 100 \mathrm{~nm}$. Ранее мы сообщали о наблюдении сверхпроводимости в пленках, выращенных магнетронным распылением [1]. В настоящей работе пленки были выращены вакуумнотермическим напылением из объемных монокристаллов $\mathrm{Cd}_{3} \mathrm{As}_{2}$ соответствующего состава.

В работах [2,3] теоретически было показано, что сверхпроводимость может наблюдаться в дираковском полуметалле $\mathrm{Cd}_{3} \mathrm{As}_{2}$. В этих работах гамильтониан электрон-электронного взаимодействия был представлен как сумма меж- и внутриорбитальной частей. Было предсказано, что если межорбитальный вклад больше, чем внутриорбитальный, то может реализоваться необычная фаза сверхпроводимости, а если меньше, то реализуется обычная сверхпроводимость. Однако природа сверхпроводимости не была изучена экспериментально и механизм сверхпроводящего спаривания не был определен.

Ранее в работе [4] сверхпроводимость наблюдалась в условиях приложения давления величиной до $13 \mathrm{GPa}$, при перестройке кристаллической структуры в $3.5 \mathrm{GPa}$ В нашей работе, так же как и в случае [1], мы наблюдали переход к сверхпроводимости без приложения внешнего давления и, соответственно, без перестройки кристаллической структуры. Также ранее в работах [5-7] методом точечного контакта (point contact) были получены результаты по поверхностной сверхпроводимости полуметаллических монокристаллов $\mathrm{Cd}_{3} \mathrm{As}_{2}$. Однако это непрямой метод, требующий вычитания больших величин, нам же удалось зафиксировать сверхпроводимость прямым методом по обращению в ноль сопротивления.

Были изготовлены и измерены три образца тонких пленок для проверки наличия воспроизводимости. Все три пленки напылялись на подложку из кремния и показали близкие значения параметров. Параметры пленок были следующие: сопротивление при комнатной температуре составляло 2034, 2135 и 2289 $\Omega$, а концентрация носителей тока, определенная с помощью холловских измерений, равнялась $3.2 \cdot 10^{18}, 3.3 \cdot 10^{18}$ и $3.1 \cdot 10^{18} \mathrm{~cm}^{-3}$ соответственно. Пленки толщиной около $100 \mathrm{~nm}$ были получены вакуумно-термическим напылением из объемных монокристаллов $\mathrm{Cd}_{3} \mathrm{As}_{2}$. Детали приготовления пленок представлены ниже.

Поликристаллические образцы $\mathrm{Cd}_{3} \mathrm{As}_{2}$ были выращены из высокочистых элементов $\mathrm{Cd}$ и $\mathrm{As}$, непосредственным сплавлением вакуумно-ампульным методом в вакуумированных $\left(\sim 10^{-2} \mathrm{~Pa}\right)$ кварцевых ампулах, покрытых пиролитическим углеродом. Синтез проводили в несколько этапов (учитывая легколетучесть элемента - As): нагрев до $620^{\circ} \mathrm{C}$ со скоростью $30^{\circ} \mathrm{C} / \mathrm{h}$ и дальнейшей выдержкой при этой температуре в течение $5 \mathrm{~h}$; затем нагрев до $780^{\circ} \mathrm{C}\left(20^{\circ} \mathrm{C} / \mathrm{h}\right)$ с выдержкой в течение 2 суток для гомогенизации расплава; охлаждение ампулы в режиме выключенной печи. Полученные поликристаллы были измельчены в порошок и исполь- 


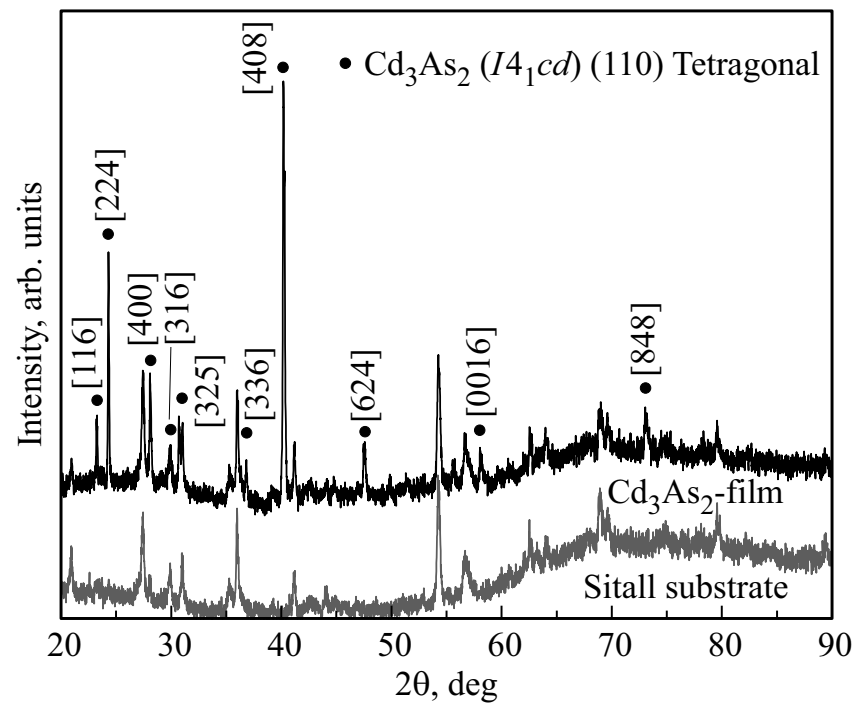

Рис. 1. Экспериментальные дифрактограммы исследуемой пленки $\mathrm{Cd}_{3} \mathrm{As}_{2}$ на подложке ситалла. Присутствующие пики подтверждают наличие только фазы $\alpha-\mathrm{Cd}_{3} \mathrm{As}_{2}$ в составе напыленной пленки.

зованы для роста монокристала в вакуумированной $\left(\sim 10^{-2} \mathrm{~Pa}\right)$ двойной кварцевой ампуле. Рост проводили в вертикальной электропечи методом направленной кристаллизации по методу Бриджмена: нагрев с постоянным вращением ампулы до температуры $735^{\circ} \mathrm{C}$ (на $15 \mathrm{~K}$ выше температуры плавления $\mathrm{Cd}_{3} \mathrm{As}_{2}$ ), выдержка расплава в течение $12 \mathrm{~h}$ и далее перемещение нижней части ампулы в холодную зону со скоростью $0.7 \mathrm{~mm} / \mathrm{h}$ при градиенте печи $1^{\circ} \mathrm{C} / \mathrm{cm}$. Анализ фазового состава полученных поликристаллов и монокристалла проводили с помощью порошкового дифрактометра. Для обоих типов кристаллов в составе присутствовала только фаза $\alpha-\mathrm{Cd}_{3} \mathrm{As}_{2}$ (пространственной группы $I 4_{1} a c d$ ).

В процессе напыления пленок давление остаточных паров в камере поддерживалось на уровне $1 \cdot 10^{-4} \mathrm{~Pa}$ и менее, предварительно был произведен отжиг вольфрамового испарителя резистивного типа, кристаллы испаряемого вещества были 1-2 mm в диаметре. В качестве подложек были использованы пластины из кремния [111], размерами $3 \times 5 \mathrm{~mm}$. Расстояние между испарителем и подложками составляло $4 \mathrm{~cm}$, температура подложек составляла $300 \mathrm{~K}$. Процесс роста пленок проводили до полного испарения материала, массу которого предварительно рассчитывали для получения пленок требуемой толщины. При массе кристалла $0.05 \mathrm{~g}$ общее время напыления составляло $8 \mathrm{~min}$. Толщина пленок составляла $100 \mathrm{~nm}$, образцы были приготовлены в виде холловских мостиков, линейные размеры которых составляли приблизительно $2 \times 0.7 \mathrm{~mm}$. Так как результаты, полученные на всех образцах, близки, ниже представлены только данные измерений на одном образце.

Для определения кристаллографической структуры пленок были приведены рентгеноструктурные (XRD) измерения на аналогичном образце, но изготовленном на подложке из ситалла. (При использовании подложки из кремния часть пиков от подложки и от пленки совпадают, что существенно затрудняет анализ рентгенограмм.) В результате, за исключением пиков, соответствующих сигналам подложки, остальные могут быть интерпретированы как пики, принадлежащие $\alpha$-фазе $\mathrm{Cd}_{3} \mathrm{As}_{2}$ пространственной группы $I 4_{1} / \mathrm{acd}$, такой же как в монокристалле (рис. 1) [8]. Эта картина соответствует дираковскому полуметаллу (DSM) [9]. Последнее также подтверждается рамановскими спектрами, в которых наблюдаются два ярких пика $\left(196 \mathrm{~cm}^{-1}\right.$ и $\left.245 \mathrm{~cm}^{-1}\right)$, полностью соответствующих положению пиков монокристалла $\mathrm{Cd}_{3} \mathrm{As}_{2}$, как представлено на рис. 2 .

Результаты рентгеновских и рамановских измерений доказывают хорошее кристаллографическое качество выращенных образцов, превышающее качество пленок, выращенных ранее методом магнетронного распыления. Высокое качество образцов также подтверждается магниторезистивными измерениями. На рис. 3 представлена температурная зависимость сопротивления для одного

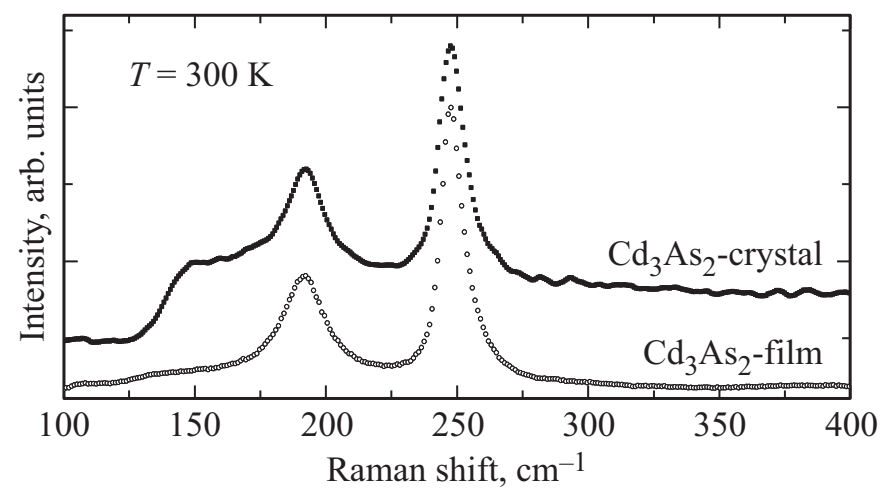

Рис. 2. На этом рисунке показан рамановский сдвиг кривых для одного из образцов $\mathrm{Cd}_{3} \mathrm{As}_{2}$ при комнатной температуре и рамановский сдвиг кривых для монокристалла $\mathrm{Cd}_{3} \mathrm{As}_{2}$ при той же температуре, который имеет характерные пики в точках $196 \mathrm{~cm}^{-1}$ и $245 \mathrm{~cm}^{-1}$.

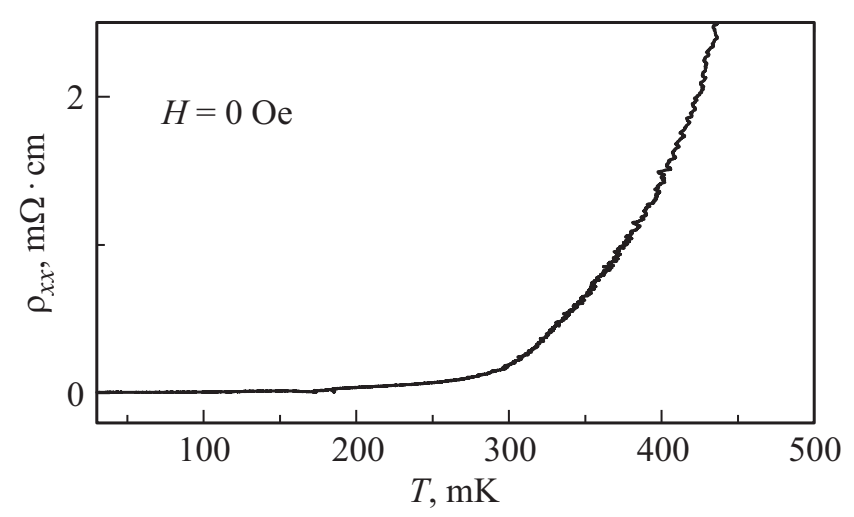

Рис. 3. Температурные зависимости электросопротивления для образца $\mathrm{Cd}_{3} \mathrm{As}_{2}$. Кривая однозначно свидельствует о наблюдавшемся переходе к сверхпроводимости. 


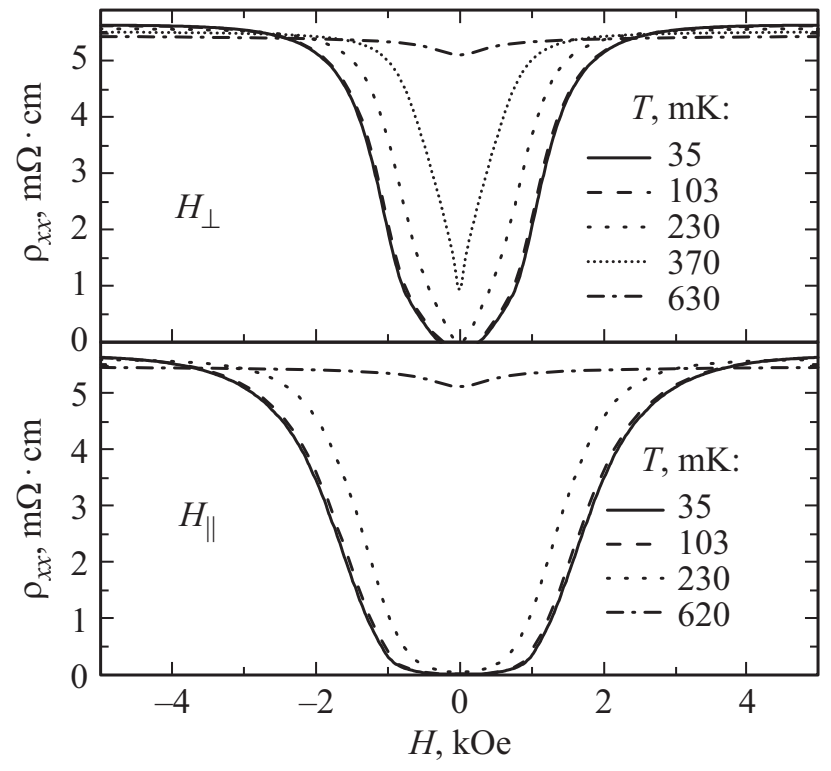

Pис. 4. Магнетополевые зависимости электросопротивления для двух направлений магнитного поля, которое было приложено в направлении $0^{\circ}$ (магнитное поле перпендикулярно плоскости образца, рис. $4, a$ ) и $90^{\circ}$ (магнитное поле вдоль плоскости образца, перпендикулярно току, рис. 4, $b$ ) в поле до 0.5 Т. Цифрами указаны температуры образца, при которых проводились измерения.

из трех образцов. В отличие от образцов, полученных магнетронным распылением и демонстрирующих разброс сопротивлений около 20-30\%, все три образца, изучаемые в настоящей работе, показывают почти идентичное поведение.

Магниторезистивные измерения проводились на установке 20 Т сверхпроводящий магнит с криостатом растворения, снабженной вращателем образца, SCM1, pacположенной в NHMFL (National High Magnetic Field Laboratory, Tallahassee, USA). На рис. 3 хорошо видны резкое падение сопротивления при температуре ниже $0.5 \mathrm{~K}$ и в дальнейшем (ниже $0.2 \mathrm{~K}$ ) обращение сопротивления в ноль, свидетельствующие о переходе образца в сверхпроводящее состояние. На рис. 4 представлены зависимости электросопротивления от магнитного поля, которое было приложено в направлении $0^{\circ}$ (магнитное поле перпендикулярно плоскости образца, рис. 4,a) и $90^{\circ}$ (магнитное поле вдоль плоскости образца, перпендикулярно току, рис. $4, b)$ в поле до 0.5 Т. Как параметр использовалась температура от $0.035 \mathrm{~K}$ до $0.630 \mathrm{~K}$.

На этих рисунках показан переход к сверхпроводимости от нормального полуметалла. Как и следовало ожидать, в случае магнитного поля, направленного перпендикулярно плоскости образца, кривые перехода значительно уже приблизительно от 0.2 до 0.015 T, если же магнитное поле лежит в плоскости образца, кривые перехода шире, $0.3-0.05$ T. Это соответствует большему значению критического поля $H_{\mathrm{c}}$, что является общим свойством для всех тонких сверхпроводящих пленок.
Оба рисунка $4, a$ и $4, b$ однозначно свидельствуют о фазовом переходе к сверхпроводимости.

Согласно нашей изначальной гипотезе, появление сверхпроводимости в наших образцах связано с влиянием подложки. Мы полагали, что сжатие подложки при охлаждении передается образцу (при уменьшении температуры), что эквивалентно тому, что образец находится под давлением, как и в случае [4]. Чтобы проверить это предположение мы исследовали пленки $\mathrm{Cd}_{3} \mathrm{As}_{2}$, выращенные на различных подложках: $\mathrm{Si}$, $\mathrm{Al}_{2} \mathrm{O}_{3}$ и ситалл. Анализ показал, что во всех случаях температурный коэффициент расширения $\mathrm{Cd}_{3} \mathrm{As}_{2}$ был больше, чем температурный коэффициент расширения подложки, иными словами образец эффективно оказывался растянут, а не сжат, и поэтому версию давления мы вынуждены были исключить. Более того, экспериментально наблюдалось, что величина температуры сверхпроводящего перехода $-T_{\mathrm{c}}$ понижалась до нуля при уменьшении температурного коэффициента расширения подложки, что противоречит предположению о сжатии образца и не позволяет применить аналогию со случаем, описанным в [4].

Мы получили диаграммы $H_{c}-T_{c}$ для исследованных образцов, показанные на рис. 5. Эти диаграммы отвечают характерному соотношению

$$
H(T)=H_{c}(0)\left[1-\left(T / T_{c}\right)^{\alpha}\right]^{\beta},
$$

где $\alpha$ и $\beta-$ коэффициенты, $H_{c}-$ критическое магнитное поле. По теории Bardeen-Cooper-Schrieffer (BCS) вблизи нулевой температуры $\alpha=2, \beta=1$. В то же время, согласно теории Гинзбурга-Ландау (ГЛ), для описания температурной зависимости вблизи $T_{c}$ и вблизи критического поля $H_{c 2}$ при рассмотрении сверхпроводимости II рода $\alpha=1$ и $\beta=1$. Однако в нашем случае в промежуточном интервале температур, то есть за рамками строгой применимости теории (ГЛ), действует та же линейная зависимость (см. рис. 5).

Таким образом, графики $H_{c}-T_{c}$ отвечают линейному поведению в промежуточном интервале температур, подобно объемным кристаллам $\mathrm{Cd}_{3} \mathrm{As}_{2}$ и $\mathrm{Bi}_{2} \mathrm{Se}_{3}$ при приложении давления $(13 \mathrm{GPa})$. Согласно теории

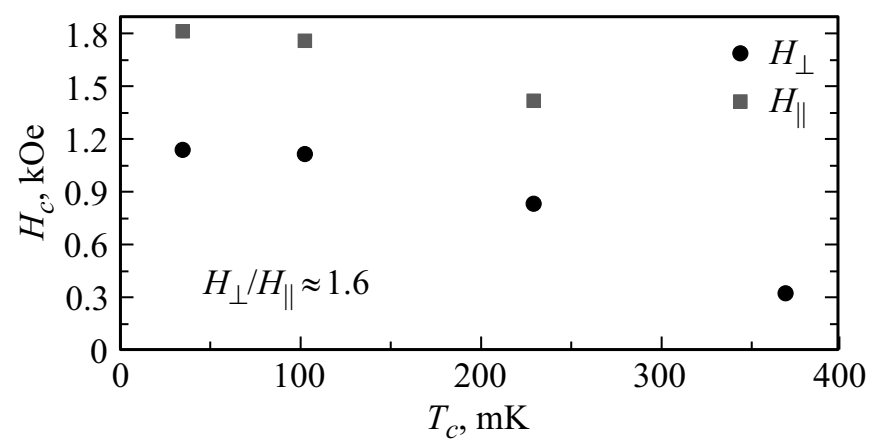

Рис. 5. Фазовая диаграмма $H_{c}-T_{c}$, построенная при $\alpha=1$ и $\beta=1$ согласно соотношению (1), что соответствует теории Гинзбурга-Ландау. 
M. Sato и Y. Ando [3], а также результатам [4-7], при давлении $13 \mathrm{GPa}$ или при поверхностной проводимости, наблюдаемой с помощью точечного контакта, наиболее вероятным является триплетное спаривание электронов. Нам остается предположить, что и в нашем случае происходит нетривиальное спаривание $p$-типа в исследованных пленках.

Таким образом, можно утверждать, что в тонких пленках дираковского полуметалла $\mathrm{Cd}_{3} \mathrm{As}_{2}$ доказано наличие сверхпроводимости в отсутствие внешнего избыточного давления.

\section{Благодарности}

Авторы благодарят К.И. Кугеля за полезные обсуждения.

\section{Финансирование работы}

Работа выполнена при поддержке проекта РНФ № 1712 01345. Национальная лаборатория сильных магнитных полей (NHMFL, USA) финансируется Национальным Научным Фондом (грант NSF/DMR-1644779) и штатом Флорида.

\section{Конфликт интересов}

Авторы заявляют, что у них нет конфликта интересов.

\section{Список литературы}

[1] A.V. Suslov, A.BDavydov, L.N. Oveshmikov, L.A. Morgun, K.I. Kugel, V.S. Zakhvalinskii, E.A. Pilyuk, A.V. Kochura, A.P. Kuzmenko, V.M. Pudalov, B.A. Aronzon. Phys. Rev. B 99, 094512 (2019).

[2] S. Kobayashi, Y. Sato. Phys. Rev. B 94, 014510 (2016).

[3] M. Sato, Y. Ando. Rep. Prog. Phys. 80, 076501 (2017).

[4] L. He, X. Hong, S. Li, Y. Jia, S. Zhang, C. Jin. npj Quantum Mater. 1, 16014 (2016).

[5] H. Wang, H. Wang, H. Liu, H. Lu, W. Yang, S. Jia, X.-J. Liu, X.C. Xie, J. Wei, J. Wang. Nature Mater. 15, 38 (2015).

[6] L. Aggarwal, A. Gaureav, G.S. Thakure, Z. Haque, A.K. Ganguli, G. Sheet. Nature Mater. 15, 32 (2015).

[7] O.O. Shvetsov, V.D. Esin, A.V. Timonina, N.N. Kolesnikiv, E.V. Deviatov. Phys. Rev. B 99, 125305 (2019). Phys. Rev. B 99, 125305 (2019).

[8] E. Arushanov. Prog. Cryst. Growth Charact. Mater. 3, 211 (1981).

[9] N.P. Armitage, E.J. Mele, A. Vishwanath. Rev. Mod. Phys. 90, 015001 (2018).

Редактор Ю.Э. Китаев 